\title{
LIE IDENTITIES ON SYMMETRIC ELEMENTS OF RESTRICTED ENVELOPING ALGEBRAS
}

\author{
SALVATORE SICILIANO AND HAMID USEFI
}

\begin{abstract}
Let $L$ be a restricted Lie algebra over a field of characteristic $p>2$ and denote by $u(L)$ its restricted enveloping algebra. We determine the conditions under which the set of symmetric elements of $u(L)$ with respect to the principal involution is Lie solvable, Lie nilpotent, or bounded Lie Engel.
\end{abstract}

\section{INTRODUCTION}

Let $A$ be an algebra with involution $*$ over a field $\mathbb{F}$. We denote by $A^{+}:=\left\{x \in A \mid x^{*}=x\right\}$ the set of symmetric elements of $A$ under $*$ and by $A^{-}:=\left\{x \in A \mid x^{*}=-x\right\}$ the set of skew-symmetric elements. A question of general interest is which properties of $A^{+}$or $A^{-}$can be lifted to the whole algebra $A$. The history of this problem goes back to Herstein [12, 13] where he had conjectured that if the symmetric or skew-symmetric elements of a ring $R$ satisfy a polynomial identity, then so does $R$. Notably this conjecture was proved by Amitsur in [1] and subsequently generalized by himself in [2].

Now consider the group algebra $\mathbb{F} G$ of a group $G$ under the canonical involution induced by $g \mapsto g^{-1}$, for every $g \in G$. There has been an intensive investigation devoted to demonstrate the extent to which the symmetric or skew-symmetric elements of $\mathbb{F} G$ under the canonical involution determine the structure of the group algebra and there has been special attention on Lie identities. In particular, the chacterization of groups $G$ for which $\mathbb{F} G^{-}$or $\mathbb{F} G^{+}$is Lie nilpotent was carried out by Giambruno, Sehgal, and Lee in [10, 11, 16]. Furthermore, if either $\mathbb{F} G^{-}$or $\mathbb{F} G^{+}$is bounded Lie Engel, and $G$ is devoid of 2-elements, Lee in [17] showed that $\mathbb{F} G$ is bounded Lie Engel. He also classified remaining groups for which $\mathbb{F} G^{+}$is bounded Lie Engel. The Lie solvable case was considered in [19, 20], however a complete answer to this case seems still under way.

Date: November 8, 2017.

2010 Mathematics Subject Classification. 16S30, 16W10, 17B50, $17 \mathrm{~B} 60$. 
Now, let $L$ be a restricted Lie algebra over a field $\mathbb{F}$ of characteristic $p>2$ and let $u(L)$ be the restricted enveloping algebra of $L$. We denote by $T$ the principal involution of $u(L)$, that is, the unique $\mathbb{F}$ antiautomorphism of $u(L)$ such that $x^{\top}=-x$ for every $x$ in $L$. We recall that $T$ is just the antipode of the $\mathbb{F}$-Hopf algebra $u(L)$.

Recently, the first author in [24] established the conditions under which $u(L)^{-}$is Lie solvable, Lie nilpotent or bounded Lie Engel.

In this paper we consider the symmetric case. Unlike the skewsymmetric case it is not clear a priori that, for example, when $u(L)^{+}$ is Lie nilpotent then $L$ is nilpotent. The symmetric elements do not form a Lie subalgebra of $u(L)$ in general (but they form a Jordan subalgebra under the Jordan bracket $\left.x \circ y=\frac{1}{2}(x y+y x)\right)$. However, despite the group ring case we present a complete answer and yet the proofs are different and more involved than the skew-symmetric case. Before stating the main results we recall the following definitions. An element $x$ of $L$ is $p$-nilpotent if $x^{[p]^{m}}=0$ for some $m \geq 1$; a subset $S$ of $L$ is $p$-nilpotent if $S^{[p]^{m}}=\left\{x^{[p]^{m}} \mid x \in S\right\}=0$ for some $m \geq 1$. The derived subalgebra of $L$ is denoted by $L^{\prime}$.

Theorem 1.1. Let $L$ be a restricted Lie algebra over a field $\mathbb{F}$ of characteristic $p>2$. Then the following conditions are equivalent:

1) $u(L)^{+}$is bounded Lie Engel;

2) $u(L)$ is bounded Lie Engel;

3) $L$ is nilpotent, $L^{\prime}$ is p-nilpotent, and $L$ contains a restricted ideal $I$ such that $L / I$ and $I^{\prime}$ are finite-dimensional.

Theorem 1.2. Let $L$ be a restricted Lie algebra over a field $\mathbb{F}$ of characteristic $p>2$. Then the following conditions are equivalent:

1) $u(L)^{+}$is Lie nilpotent;

2) $u(L)$ is Lie nilpotent;

3) $L$ is nilpotent and $L^{\prime}$ is finite-dimensional and p-nilpotent.

Theorem 1.3. Let $L$ be a restricted Lie algebra over a field $\mathbb{F}$ of characteristic $p>2$. Then the following conditions are equivalent:

1) $u(L)^{+}$is Lie solvable;

2) $u(L)$ is Lie solvable;

3) $L^{\prime}$ is finite-dimensional and p-nilpotent.

The equivalence of 2) and 3) in Theorems 1.1-1.3 was established in [23. Our main contribution is to prove that 2) implies 3) in these theorems. In combination with [24], we can conclude that, in odd characteristic, if either $u(L)^{+}$or $u(L)^{-}$is Lie solvable (respectively, bounded Lie Engel or Lie nilpotent) then so is the whole algebra $u(L)$. Such conclusions are no longer true in characteristic 2. 
Finally, let $L$ be an arbitrary Lie algebra over a field of characteristic different from 2 and denote by $U(L)$ the ordinary enveloping algebra of $L$. A further consequence of our main results is that $U(L)^{+}$(under the principal involution) is Lie solvable or bounded Lie Engel only when $L$ is abelian.

\section{Preliminaries}

Throughout the paper all restricted Lie algebras are defined over a field $\mathbb{F}$ of characteristic $p>2$.

Let $A$ be an associative algebra over $\mathbb{F}$ with an involution. The Lie bracket on $A$ is defined by $[x, y]=x y-y x$, for every $x, y \in$ $A$. Longer Lie commutators of $A$ are recursively defined as follows: $\left[x_{1}, x_{2}, \ldots, x_{n+1}\right]=\left[\left[x_{1}, x_{2}, \ldots, x_{n}\right], x_{n+1}\right]$ and $\left[x_{,_{n+1}} y\right]=\left[\left[x,_{n} y\right], y\right] . \mathrm{A}$ subset $S$ of $A$ is said to be Lie nilpotent if there exists a positive integer $n$ such that

$$
\left[x_{1}, \ldots, x_{n}\right]=0
$$

for every $x_{1}, \ldots, x_{n} \in S$, while $S$ is said to be bounded Lie Engel if there is an $n$ such that

$$
[x, n y]=0
$$

for every $x, y \in S$. Also, following [18], we put $\left[x_{1}, x_{2}\right]^{o}=\left[x_{1}, x_{2}\right]$ and

$$
\left[x_{1}, x_{2}, \ldots, x_{2^{n+1}}\right]^{o}=\left[\left[x_{1}, \ldots, x_{2^{n}}\right]^{o},\left[x_{2^{n}+1}, \ldots, x_{2^{n+1}}\right]^{o}\right] .
$$

The subspace spanned by all $\left[a_{1}, \ldots, a_{2^{n}}\right]^{o}$, where $a_{i} \in S$, is denoted by $\delta_{n}(S)$. The subset $S$ is said to be Lie solvable if there exists an $n$ such that $\delta_{n}(S)=0$.

Moreover, we denote by $\gamma_{n}(L)(n \geq 1)$ and $\delta_{n}(L)(n \geq 0)$ the terms of the lower central series and derived series of $L$, respectively. Also, for a subset $S$ of $L$ we denote by $S_{p}$ the restricted subalgebra generated by $S$. A polynomial of the form $x^{p^{k}}+\alpha_{1} x^{p^{k-1}}+\cdots+\alpha_{k} x \in \mathbb{F}[x]$ is called a $p$-polynomial. An element $x$ of $L$ is said to be $p$-algebraic if $\operatorname{dim}_{\mathbb{F}}\langle x\rangle_{p}<\infty$. Finally, $Z(L)$ is the center of $L$.

Theorem 2.1 ([1]). Let $A$ be an associative algebra with involution. If $A^{+}$(or $\left.A^{-}\right)$satisfies a polynomial identity then $A$ satisfies a polynomial identity.

Restricted Lie algebras whose enveloping algebras satisfy polynomial identities are characterized by Passman [21] and Petrogradski [22]. A theorem of Kukin (see [15, §4, Lemma 1.10]) states that any restricted subalgebra $H$ of $L$ of finite codimension contains an ideal of finite codimension in $L$. Combining these results we get the following: 
Theorem 2.2. Let $L$ be a restricted Lie algebra over a field of characteristic $p>0$. Then $u(L)$ satisfies a polynomial identity if and only if $L$ has a restricted ideal $A$ of finite codimension in $L$ such that $A$ is nilpotent of class two and $A^{\prime}$ is finite-dimensional and p-nilpotent.

Theorem 2.3 ([25]). Let $L$ be a Lie algebra with a nilpotent ideal $M$ such that $L / M^{\prime}$ is nilpotent. Then $L$ is nilpotent.

Corollary 2.4. Let $L$ be a restricted Lie algebra with a nilpotent ideal $M$ such that $L /\left(M^{\prime}\right)_{p}$ is nilpotent. Then $L$ is nilpotent.

Proof. Suppose that $\gamma_{c+1}(L) \subseteq\left(M^{\prime}\right)_{p}$. Then $\gamma_{c+2}(L) \subseteq\left[\left(M^{\prime}\right)_{p}, L\right] \subseteq M^{\prime}$. Thus, $L / M^{\prime}$ is a nilpotent Lie algebra and it follows from Theorem 2.3 that $L$ is nilpotent.

Lemma 2.5. Let $L$ be a restricted Lie algebra such that $u(L)^{+}$is Lie solvable. If $Z(L)$ is infinite-dimensional, then $u(L)$ is Lie solvable.

Proof. The proofs of Lemma 1 and Corollary 1 in [24] work in the symmetric situation as well (by using $x_{i}+x_{i}^{*}$ instead of $x_{i}-x_{i}^{*}$ ).

A similar conclusion also holds for the Lie nilpotence case. Now suppose that $u(L)^{+}$is Lie solvable and $L$ is nilpotent of class $c$, say. If $\gamma_{c}(L)_{p}$ is infinite-dimensional, then because $\gamma_{c}(L)_{p}$ is a central ideal of $L$, we deduce by Lemma 2.5 that $u(L)$ itself is Lie solvable. But then $L^{\prime}$ is finite-dimensional and $p$-nilpotent, see [23, Theorem 1.3] or [28, Lemma 4.4]. This contradiction yields the following:

Corollary 2.6. Let $L$ be a nilpotent restricted Lie algebra of class $c \geq 2$. If $u(L)^{+}$is Lie solvable, then $\gamma_{c}(L)_{p}$ is finite-dimensional.

The following can be deduced from classical results. For the proof see, for example, [23] or [28].

Lemma 2.7. If $u(L)$ is Lie solvable then $L^{\prime}$ is p-nilpotent.

In the sequel, we shall freely use the following fact.

Remark 2.8. Let $A$ be an algebra over a field of characteristic not 2 with involution $*$. If $J$ is a $*$-invariant ideal of $A$ then $(A / J)^{+}$with respect to the induced involution coincides with the image of $A^{+}$under the canonical map $A \rightarrow A / J$. In particular, this applies to the case where $A=u(L)$ and $J$ is the associative ideal generated by a restricted ideal of $L$. 


\section{Proofs of the main Results}

Lemma 3.1. Let $L=\langle H\rangle_{p}$ where $H$ is a finite-dimensional Lie algebra. If $u(L)^{+}$is bounded Lie Engel then $L$ is nilpotent.

Proof. Clearly, it is enough to show that $H$ is nilpotent. By Engel's Theorem it suffices to prove that ad $x$ is nilpotent, for every $x \in H$. Suppose to the contrary that ad $x$ is not nilpotent, for some $x \in H$. Let $\overline{\mathbb{F}}$ be the algebraic closure of $\mathbb{F}$. Since $\operatorname{ad}(x \otimes 1)$ is not a nilpotent operator, there exists a nonzero $b \in H \otimes \overline{\mathbb{F}}$ such that $[x \otimes 1, b]=\alpha b$, where $\alpha$ is a non-zero eigenvalue of $\operatorname{ad}(x \otimes 1)$. We may replace $x$ with $\alpha^{-1} x$ to assume that $[x \otimes 1, b]=b$. Let $b=\sum_{k=1}^{t} b_{k} \otimes \alpha_{k}$, where $b_{k} \in H$ and $\alpha_{k} \in \mathbb{F}$. Then

$$
b^{2}=\sum_{1 \leq i<j \leq t}\left(b_{i} b_{j}+b_{j} b_{i}\right) \otimes \alpha_{i} \alpha_{j}+\sum_{k=1}^{t} b_{k}^{2} \otimes \alpha_{k}^{2} .
$$

Note that $b_{i} b_{j}+b_{j} b_{i}, x^{2} \in u(L)^{+}$. There exists an integer $m$ such that

$$
\left[b_{i} b_{j}+b_{j} b_{i}, p^{m} x^{2}\right]=0
$$

for all $i, j$. Similarly, $\left[b_{k}^{2}, p^{m} x^{2}\right]=0$, for every $k$. It follows that

$$
\left[b^{2}, p^{m} x^{2} \otimes 1\right]=\sum_{1 \leq i<j \leq t}\left[\left(b_{i} b_{j}+b_{j} b_{i}\right),_{p^{m}} x^{2}\right] \otimes \alpha_{i} \alpha_{j}+\sum_{k=1}^{t}\left[b_{k}^{2}, p^{m} x^{2}\right] \otimes \alpha_{k}^{2}=0 .
$$

On the other hand, we have

$$
\left[x^{2} \otimes 1, b^{2}\right]=\left[(x \otimes 1)^{2}, b^{2}\right]=4 b^{2}((x+1) \otimes 1) .
$$

Thus,

$$
\left[b^{2}{ }_{p^{m}} \frac{x^{2} \otimes 1}{4}\right]=-b^{2}\left((x+1)^{p^{m}} \otimes 1\right)=-b^{2}(x \otimes 1)^{p^{m}}-b^{2}=0
$$

By the PBW Theorem for restricted Lie algebras (see e.g. [27, §2, Theorem 5.1]) this is possible only if $x$ is $p$-nilpotent which implies that ad $x$ is nilpotent, a contradiction.

Lemma 3.2. Let $L$ be a metabelian restricted Lie algebra containing an abelian ideal $N$ of finite codimension. If $u(L)^{+}$is bounded Lie Engel, then $L$ is nilpotent.

Proof. We argue by induction on $\operatorname{dim} L / N$. Note that $L / N$ is nilpotent, by Lemma 3.1. Let $M=N+\left(L^{\prime}\right)_{p}$. Then $M$ is metabelian and since $L / N$ is nilpotent, we have $\operatorname{dim} M / N<\operatorname{dim} L / N$. Thus, by induction on $\operatorname{dim} L / N$, we can assume that $M$ is nilpotent. In order to prove $L$ is nilpotent, it is enough, by Corollary 2.4, to show that $L /\left(M^{\prime}\right)_{p}$ is nilpotent. So we replace $L$ with $L /\left(M^{\prime}\right)_{p}$ to assume that $\left[L^{\prime}, N\right]=0$. 
We also replace $N$ with $N+\left(L^{\prime}\right)_{p}$ to assume that $L^{\prime} \subseteq N$. Now let $x \in L \backslash N$. Since $L / N$ is finite-dimensional, there exists a $p$-polynomial $f$ such that $f(x) \in N$. It is easy to see that, for every $a \in N$, the Lie algebra $H$ generated by $x$ and $a$ is of dimension at most $\operatorname{deg}(f)+1$. So, by Lemma 3.1, $H_{p}$ is nilpotent. Indeed, the nilpotency class of $H_{p}$ depends only on $\operatorname{dim} H$. We deduce that $\left[N,_{m} x\right]=0$, where $m$ depends only on $x$. Hence the restricted subalgebra $I$ generated by $N$ and $x$ is nilpotent. Note that $I$ is in fact a restricted ideal of $L$. By Corollary 2.4, we can replace $L$ with $L /\left(I^{\prime}\right)_{p}$. But then $\operatorname{dim} L / I<\operatorname{dim} L / N$. So, by the induction hypothesis, $L$ is nilpotent, as required.

Proposition 3.3. Let $L$ be a restricted Lie algebra such that $u(L)^{+}$is bounded Lie Engel. Then L is nilpotent.

Proof. It follows from Theorem 2.1 that $u(L)$ is PI. Thus, by Theorem 2.2, there exists a nilpotent restricted ideal $N$ of $L$ of finite codimension. Hence, by Corollary [2.4, it is enough to prove that $L /\left(N^{\prime}\right)_{p}$ is nilpotent. Thus, we can assume that $L$ has an abelian ideal $N$ of finite codimension. Since $L / N$ is finite-dimensional, by Lemma 3.1 we see that $L / N$ is nilpotent. Thus $L$ is solvable. Let $d$ be the derived length of $L$ and consider $M=\delta_{d-2}(L)_{p}$. By Lemma 3.2, we know that $M$ is nilpotent. Moreover, by induction on the derived length we have that $L /\left(M^{\prime}\right)_{p}=L / \delta_{d-1}(L)_{p}$ is nilpotent. Therefore, by Corollary 2.4, we conclude that $L$ is nilpotent.

Corollary 3.4. Let $L$ be a restricted Lie algebra. The symmetric elements of $u(L)$ commute if and only if $L$ is abelian.

Proof. The sufficiency is obvious. Suppose $u(L)^{+}$is commutative and that, if possible, $L$ is not abelian. Since, by Proposition 3.3 , $L$ is nilpotent, we can find non-commuting elements $x, y \in L$ such that $z=[x, y]$ is central. But then $x^{2}$ and $2 x y-z$ are symmetric elements of $u(L)$ and yet $\left[x^{2}, 2 x y-z\right]=4 x^{2} z$ is not zero, a contradiction.

Proposition 3.5. Let $L$ be a restricted Lie algebra such that $u(L)^{+}$is bounded Lie Engel. Then $L^{\prime}$ is p-nilpotent.

Proof. By hypothesis, there exists an $m$ such that $\left[a,_{m} b\right]=0$ for every $a, b \in u(L)^{+}$. Suppose, if possible, that $L^{\prime}$ is not $p$-nilpotent. By Proposition 3.3 we know $L$ is nilpotent, hence there exists a minimal $n>2$ such that $\gamma_{n}(L)_{p}$ is $p$-nilpotent. Put $\mathfrak{L}:=L / \gamma_{n}(L)_{p}$. Let $t$ be an integer such that $p^{t} \geq m$. Then from the assumption it follows that there exist $x, y \in \mathfrak{L}$ such that $z=[x, y] \in Z(\mathfrak{L})$ and $z^{[p]^{t}} \neq 0$. Clearly, 
the element $x^{2}$ and $2 x y-z$ of $u(\mathfrak{L})$ are symmetric. Moreover, it can be seen by an easy induction that for every $r>0$ one has

$$
\left[x^{2}{ }_{r} 2 x y-z\right]=4^{r} x^{2} z^{r} .
$$

Since $p^{t} \geq m$ and $p \neq 2$, from relation (1) we deduce that $x^{2} z^{p^{t}}=0$. On the other hand, since $x$ and $z^{[p]^{t}}$ are $\mathbb{F}$-linearly independent (as $z^{[p]^{t}}$ is central whereas $x$ is not), the last conclusion contradicts the PBW Theorem, completing the proof.

It is now a simple matter to prove Theorems 1.1 and 1.2 .

Proof of Theorem 1.1. Of course, in view of Theorem 2 of [23], it is enough to show that 1) implies 3). By Lemmas 3.3 and 3.5, we already know that $L$ is nilpotent and $L^{\prime}$ is $p$-nilpotent. Moreover, by Theorem 2.1. $u(L)$ satisfies a polynomial identity and then, by Theorem $[2.2, L$ contains a restricted ideal $I$ such that $L / I$ and $I^{\prime}$ are finite-dimensional. This finishes the proof.

Proof of Theorem 1.2. It is enough, by Theorem 1 of [23], to show that 1) implies 3). So, suppose $u(L)^{+}$is Lie nilpotent. Then, by Theorem 1.1, we just need to show that $L^{\prime}$ is finite-dimensional. By Theorem 1.1. we know that $L$ is nilpotent of class $c$, say. Now, by Corollary 2.6. $\gamma_{c}(L)_{p}$ is finite-dimensional. We can proceed by induction on $c$ to conclude that $L^{\prime}+\gamma_{c}(L)_{p} / \gamma_{c}(L)_{p}$ is finite-dimensional, hence $L^{\prime}$ is finite-dimensional, too.

Proposition 3.6. Let $L$ be a restricted Lie algebra such that $u(L)^{+}$is Lie solvable. Then $L$ is solvable.

Proof. We can suppose, without loss of generality, that the ground field $\mathbb{F}$ is algebraically closed. Moreover, by Theorem 2.1, $u(L)$ is PI and then, by Theorem 2.2, there exists a nilpotent restricted ideal $N$ of $L$ of finite codimension. Thus we can replace $L$ by $L / N$ and assume that $L$ is finite-dimensional. Suppose that $L$ is a counterexample of minimal dimension. Now, if $M$ is a non-trivial restricted ideal of $L$, then $u(J)^{+}$ and $u(L / M)^{+}$are Lie solvable. Hence the minimality of $L$ forces that both $M$ and $L / M$ are solvable, so that $L$ is solvable, a contradiction. Consequently, $L$ has no non-trivial restricted ideal. Now, we claim that $L^{\prime}$ is a simple Lie algebra. Let $I$ be a nonzero ideal of $L^{\prime}$. As $\left(L^{\prime}\right)_{p}$ is a nonzero restricted ideal of $L$ we have $\left(L^{\prime}\right)_{p}=L$ and then $I$ is an ideal of $L$. It follows that $I_{p}=L$ and then $L^{\prime}=\left[I_{p}, I_{p}\right]=[I, I] \subseteq I$, so that $I=L^{\prime}$, as claimed. In particular, $L^{\prime}$ is a simple restricted $L$-module. Denote by $\mathcal{J}$ the Jacobson radical of $u(L)$. Note that the simple modules of $u(L)$ and $u(L) / \mathcal{J}$ are the same. Since $\mathcal{J}$ is $\top$-invariant we have that $(u(L) / \mathcal{J})^{+}$under the induced involution is 
Lie solvable. As a consequence, if $u(L) / \mathcal{J} \cong \oplus_{i=1}^{r} M_{n_{i}}(\mathbb{F})$ is the the Wedderburn decomposition of $u(L) / \mathcal{J}$, in view of Lemma 3.2 of [19] we have that $n_{i} \leq 2$ for every $i$. Therefore every irreducible representation of $u(L)$ has degree 1 or 2 . In particular $L^{\prime}$ has dimension less than 3 , so that $L$ is solvable, a contradiction.

Lemma 3.7. Let $L$ be a finite-dimensional restricted Lie algebra such that $u(L)^{+}$is Lie solvable. Then $L^{\prime}$ is p-nilpotent.

Proof. By Proposition 3.6, we know that $L$ is solvable. By induction on the derived length of $L$, we can assume $L$ is metabelian. Furthermore, we can suppose that the ground field $\mathbb{F}$ is algebraically closed. Let $\mathcal{J}$ be the Jacobson radical of $u(L)$ and consider the restricted Lie algebra $H=L+\mathcal{J} / \mathcal{J}$. Since $\mathcal{J}$ is a nilpotent ideal of $u(L)$, it suffices to show that $H^{\prime}$ is $p$-nilpotent. Thus, we may assume that $H$ is not abelian. Note that $u(L) / \mathcal{J} \cong \mathbb{F} \oplus \cdots \mathbb{F} \oplus M_{2}(\mathbb{F}) \oplus \cdots \oplus M_{2}(\mathbb{F})$, by [19]. Without loss of generality we can assume that $H$ is a restricted Lie subalgebra of $M_{2}(\mathbb{F})$. Since $M_{2}(\mathbb{F})$ is not Lie metabelian, we have $\operatorname{dim} H \leq 3$. If $\operatorname{dim} H=2$, then there exists $x, y \in H$ such that with $[x, y]=x$. In this case we must have $x^{p}=0$ and $y^{p}=y$, so we are done. Assume then $\operatorname{dim} H=3$. It is clear that the identity matrix $I_{2}$ must be in $H$, otherwise $M_{2}(\mathbb{F})$ is spanned by $H$ and $I_{2}$ so that $M_{2}(\mathbb{F})$ is Lie metabelian, which is not possible. Note that $H^{\prime} \subseteq\left[M_{2}(\mathbb{F}), M_{2}(\mathbb{F})\right]=\operatorname{sl}_{2}(\mathbb{F})$. Since $H^{\prime}$ is abelian and $\operatorname{sl}_{2}(\mathbb{F})$ has no 2-dimensional abelian subalgebra, it follows that $\operatorname{dim} H^{\prime}=1$. Let $x \in H^{\prime} \subseteq \operatorname{sl}_{2}(\mathbb{F})$. Since $\operatorname{tr}(x)=0$, it follows that $x$ and $I_{2}$ are linearly independent. Let $y \in H$ so that $x, y$ and $I_{2}$ span $H$. Since $H$ is not abelian, we can assume $[x, y]=x$. But then $x^{p}$ is a central element in $H$ and so $x^{p}=\alpha I_{2}$, for some $\alpha \in \mathbb{F}$. Since $x \in \operatorname{sl}_{2}(\mathbb{F})$ and $p \geq 3$, $\operatorname{tr}\left(x^{p}\right)=0$. Thus, $\alpha=0$ and so $x^{p}=0$.

Lemma 3.8. Let $L$ be a nilpotent restricted Lie algebra such that $u(L)^{+}$ is Lie solvable. Then $L^{\prime}$ is finite-dimensional and p-nilpotent.

Proof. Let $c$ be the nilpotence class of $L$. First we prove that $\gamma_{c}(L)$ is $p$-nil. So let $x \in L$ and $y \in \gamma_{c-1}(L)$ and let $H$ be the restricted subalgebra of $L$ generated by $x$ and $y$. Let $\mathcal{J}$ be the Jacobson radical of $u(H)$. By the Razmyslov-Kemer-Braun Theorem (see e.g. [7]) $\mathcal{J}$ is a nilpotent ideal of $u(H)$. Note that $x^{2}, y^{2}$ and $u=2 x y-z$ are symmetric. In $u(H)$ we have $[x, 2 x y-z]=2 x z$. So, $\left[x^{2}, u\right]=4 x^{2} z$. We can similarly deduce that, $\left[y^{2}, u\right]=-4 y^{2} z$. Thus,

$$
\left[\left[x^{2}, u\right],\left[y^{2}, u\right]\right]=16\left[x^{2} z, y^{2} z\right]=-64 x y z^{3}+32 z^{4} \text {. }
$$


By Proposition 2.6 of [19], $(u(H) / \mathcal{J})^{+}$is Lie metabelian. It follows that $2 x y z^{3}-z^{4} \in \mathcal{J}$. Note that

$$
\left[\left[x, 2 x y z^{3}-z^{4}\right], y\right]=2 z^{5} \in \mathcal{J} .
$$

Thus, there exists an integer $k$ such that

$$
\left(z^{p^{k}}\right)^{5}=0
$$

which implies that $z$ must be $p$-nilpotent. Note that $\gamma_{c}(L)_{p}$ is finitedimensional, by Corollary [2.6. Now we consider $L / \gamma_{c}(L)_{p}$. By induction on $c$, we have that $L^{\prime}+\gamma_{c}(L)_{p} / \gamma_{c}(L)_{p}$ is finite-dimensional and $p$-nilpotent. Combining with the fact that $\gamma_{c}(L)_{p}$ is also finitedimensional and $p$-nilpotent yields the required result.

Lemma 3.9. Let $L$ be a metabelian restricted Lie algebra such that $u(L)^{+}$is Lie solvable. Then the space of fixed points of the action of ad $x$ on $L^{\prime}$ is finite-dimensional, for every $x \in L$.

Proof. Let $V$ be the subspace of $L^{\prime}$ consisting of the fixed points of the action of ad $x$ on $L^{\prime}$. Note that for every $r \geq 1$ and $a_{1}, \ldots, a_{r} \in V$, we have

$$
\left[x, a_{1} \cdots a_{r}\right]=r a_{1} \cdots a_{r} .
$$

Thus, for every odd number $k$ and $a_{1}, \ldots, a_{k} \in V$, we have

$$
2 x a_{1} \cdots a_{k}-k a_{1} \cdots a_{k}=x a_{1} \cdots a_{k}+\left(x a_{1} \cdots a_{k}\right)^{\top} \in u(L)^{+} .
$$

Let $n$ be a positive integer and let $m_{1}=2^{n+1}+2^{n}-2, m_{2}=2^{n+1}+2^{n}$ and $m_{3}=2^{n+1}+2^{n}+2$. By the PBW Theorem, it is enough to show that for every $a_{1}, \ldots, a_{m_{i}} \in V$ there exists $\alpha \in \mathbb{F}$ such that

$$
x a_{1} a_{2} \cdots a_{m_{i}}+\alpha a_{1} a_{2} \cdots a_{m_{i}} \in \delta_{n}\left(u(L)^{+}\right),
$$

for $i=1,2,3$. Using Equation (2), the claim can be easily checked for $n=0$. Now we assume that $n \geq 1$ and we prove the induction step for $m_{1}$. So set $r=2^{n}+2^{n-1}-2, s=2^{n}+2^{n-1}$ and let $a_{1}, \ldots, a_{r}, b_{1}, \ldots, b_{s} \in$ $V$. By the induction hypothesis we have

$$
\begin{aligned}
& u=x a_{1} \cdots a_{r}+\alpha a_{1} \cdots a_{r} \in \delta_{n}\left(u(L)^{+}\right), \\
& v=x b_{1} \cdots b_{s}+\beta b_{1} \cdots b_{s} \in \delta_{n}\left(u(L)^{+}\right),
\end{aligned}
$$

for some $\alpha, \beta \in \mathbb{F}$. Thus,

$$
[u, v]=2 x a_{1} \cdots a_{r} b_{1} \cdots b_{s}+(\beta s-\alpha r) a_{1} \cdots a_{r} b_{1} \cdots b_{s} \in \delta_{n+1}\left(u(L)^{+}\right) .
$$

Similarly, for $m_{2}$ we take $r=2^{n}+2^{n-1}-2$ and $s=2^{n}+2^{n-1}+2$ and for $m_{3}$ we take $r=2^{n}+2^{n-1}$ and $s=2^{n}+2^{n-1}+2$ and argue the same way to establish the inductive step. 
Lemma 3.10. Let $L=\langle y\rangle_{p}+N$ where $N$ is an abelian restricted ideal of finite codimension in L. If $u(L)^{+}$is Lie solvable, then $L^{\prime}$ is finitedimensional.

Proof. Without loss of generality we can suppose the ground field $\mathbb{F}$ is algebraically closed. We argue by induction on $\operatorname{dim} L / N$. By [27, $\S 2$, Theorem 3.6], there exists a $p$-polynomial $g$ such that $x=g(y)$ lies in $L \backslash N$ and satisfies either $x^{[p]}=0(\bmod N)$ or $x^{[p]}=x(\bmod$ $N)$. Thus, $(\operatorname{ad} x)^{p}=0$ or $(\operatorname{ad} x)^{p}=\operatorname{ad} x$ in $L$. Let $H=\langle x, N\rangle_{p}$. We claim that $\left(H^{\prime}\right)_{p}$ is finite-dimensional. If $(\operatorname{ad} x)^{p}=0$, then $H$ is nilpotent and the claim follows from Lemma 3.8. On the other hand, if $(\operatorname{ad} x)^{p}=\operatorname{ad} x$, since $H^{\prime}=[x, H]$ then $(\operatorname{ad} x)^{p-1}$ acts the identity on $H^{\prime}$. Therefore all the eigenvalues of ad $x$ on $H^{\prime}$ are $(p-1)^{\text {th }}$ root of unity and $H^{\prime}$ decomposes as the sum of the relative eigenspaces. Now, let $\lambda$ be an eigenvalue of ad $x$ and put $\bar{x}=\lambda^{-1} x$. Then, by Lemma 3.9, the vector space of fixed points of ad $\bar{x}$ on $H^{\prime}$ is finitedimensional, which is equivalent to saying that the eigenspace relative to the eigenvalue $\lambda$ of ad $x$ is finite-dimensional. Hence, $H^{\prime}$ is finitedimensional. If there exists an element $u \in H^{\prime}$ such that $\langle u\rangle_{p}$ is not finite-dimensional, then the center of $H$ would be infinite-dimentional. This implies, by Lemma 2.6, that $u(H)$ is Lie solvable and in turn, by Lemma 2.7, $H^{\prime}$ is $p$-nilpotent, a contradiction. We conclude that $\left(H^{\prime}\right)_{p}$ is finite-dimensional, as claimed. Now we replace $L$ with $L /\left(H^{\prime}\right)_{p}$ to assume that $H$ is abelian. But then $L$ has an abelian restricted ideal $H$ of smaller codimension than $N$. Therefore it follows by induction that $L^{\prime}$ is finite-dimensional.

Proof of Theorem [1.3. By Theorem 3 of [23], it is enough to show that 1) implies 3). Note that, by Proposition 3.6, $L$ is solvable. Hence, by induction on the derived length, we can assume that $L$ is metabelian. By Theorem 2.1, $u(L)$ is PI. Thus, by Theorem 2.2, there exists a nilpotent restricted ideal $N$ of $L$ of finite codimension. By Lemma 3.8, $N^{\prime}$ is finite-dimensional and $p$-nilpotent. So we can replace $L$ with $L /\left(N^{\prime}\right)_{p}$ to assume that $N$ is abelian. Now we argue by induction on $\operatorname{dim} L / N$. Let $I$ be the restricted ideal generated by $L^{\prime}+N$. Since $L$ is metabelian, it follows that $I$ is nilpotent of class two. Thus again, by Lemma 3.8, we can replace $L$ with $L /\left(I^{\prime}\right)_{p}$ to assume that $\left[N, L^{\prime}\right]=0$. Now let $x \in L \backslash N$ and denote by $H$ the restricted subalgebra generated by $x$ and $L^{\prime}+N$. By Lemma 3.10, $H^{\prime}$ is finite-dimensional. We claim that $H^{\prime}$ is $p$-nilpotent. If $L^{\prime}+N$ has an element which is not $p$-algebraic then $Z(H)$ is infinite-dimensional and it follows from Lemma 2.6 that $u(H)$ is Lie solvable which in turn implies, by Lemma 2.7, that $H^{\prime}$ is $p$-nilpotent. Hence we can assume that every element of $L^{\prime}+N$ 
is $p$-algebraic. Since $\operatorname{dim} L / N$ is finite, there exists a $p$-polynomial such that $z=f(x) \in N$. Thus $z$ is $p$-algebraic and so is $x$. Now let $y \in L^{\prime}+N$. Then note that $M=\langle x, y\rangle_{p}$ is finite-dimensional. Hence, by Lemma 3.7. $M^{\prime}$ is $p$-nilpotent. We just showed that $[x, y]$ is $p$-nilpotent for every $y \in L^{\prime}+N$. Thus, $H^{\prime}$ is $p$-nilpotent. Now we can replace $L$ with $L /\left(H^{\prime}\right)_{p}$. So, $H$ is an abelian restricted ideal of $L$ and $\operatorname{dim} L / H<\operatorname{dim} L / N$. Thus, by induction hypothesis, $L^{\prime}$ is finite-dimensional and $p$-nilpotent, as required

\section{CONCLUding REMARKS}

Note that if $A$ is any algebra with involution then $\left[A^{+}, A^{+}\right] \subseteq A^{-}$. In particular, if $A^{-}$is Lie solvable then so is $A^{+}$. Therefore, Theorem 1.3 generalizes Theorem 1 of [24]. Moreover, since in characteristic 2 the sets of symmetric and skew-symmetric elements obviously coincide, Examples 1 and 2 of [24] show that the assumption $\operatorname{char}(\mathbb{F}) \neq 2$ in Theorems 1.1, 1.2, and 1.3 cannot be removed.

Finally, let $L$ be an arbitrary Lie algebra over a field $\mathbb{F}$ of characteristic $p \neq 2$ and denote by $U(L)$ the ordinary universal enveloping algebra of $L$. Suppose that $U(L)^{+}$(with respect to the principal involution) is Lie solvable or bounded Lie Engel. If $p=0$ then, by Theorem 2.1, $U(L)$ satisfies a polynomial identity. Thus, $L$ is abelian by a theorem of Latysěv (see e.g [3, §6.7, Theorem 25]). Now suppose that $p>2$ and consider the universal $p$-envelope of $L$

$$
\hat{L}:=\sum_{k \geq 0} L^{p^{k}} \subseteq U(L)
$$

where $L^{p^{k}}$ is the $\mathbb{F}$-vector space spanned by the set $\left\{l^{p^{k}} \mid l \in L\right\}$. Then $\hat{L}$ is a restricted Lie algebra with $p$-map given by $h^{[p]}=h^{p}$ for all $h \in \hat{L}$. Note that by Corollary 1.1.4 of [26], $U(L)=u(\hat{L})$. So, Theorem 1.3 and Theorem 1.1 entail that $(\hat{L})^{\prime}$ is $p$-nilpotent. Since $u(\hat{L})=U(L)$ has no nontrivial zero divisors, we conclude that $L^{\prime}=0$.

We have proved

Corollary 4.1. Let $L$ be a Lie algebra over a field $\mathbb{F}$ of characteristic $p \neq 2$. Then $U(L)^{+}$is Lie solvable or bounded Lie Engel if and only if $L$ is abelian.

\section{ACKNOWLEDGMENTS}

The second author would like to thank the Department of Matematics of the University of Salento for its hospitality during his visit while this work was completed. 


\section{REFERENCES}

[1] S.A. Amitsur: Rings with involution. Israel J. Math. 6 1968 99-106.

[2] S.A. Amitsur: Identities in rings with involutions, Israel J. Math. 7 (1969), 63-68.

[3] Yu.A. Bahturin: Identical relations in Lie algebras. VNU Science Press, Utrecht, 1987.

[4] Yu.A. Bahturin, A.A. Mikhalev, V.M. Petrogradsky, M.V. Zaicev: Infinitedimensional Lie superalgebras. de Gruyter Expositions in Mathematics, 7. Walter de Gruyter \& Co., Berlin, 1992.

[5] V. Bovdi: On symmetric units in group algebras, Comm. Algebra 29 (2001), 5411-5422.

[6] V. Bovdi, L.G. Kovács, S.K. Sehgal: Symmetric units in modular group algebras, Comm. Algebra 24 (1996), 803-808.

[7] A. Braun: The nilpotency of the radical in a finitely generated PI ring, J. Algebra 89 (1984), 375-396.

[8] A. Giambruno, C. Polcino Milies, S.K. Sehgal: Lie properties of symmetric elements in group rings, J. Algebra 321 (2009), 890-902.

[9] A. Giambruno, C. Polcino Milies, S.K. Sehgal: Group identities on symmetric units, J. Algebra 322 (2009), 2801-2815.

[10] A. Giambruno, S.K. Sehgal: Lie nilpotence of group rings, Comm. Alg. 21 (1993), 4253-4261.

[11] A. Giambruno, S.K. Sehgal: Group algebras whose Lie algebra of skewsymmetric elements is nilpotent, In: Group, Rings and algebras. Contemp. Math 420, Amer. Math. Soc. Providence, RI 2006, pp. 113-120.

[12] I.N. Herstein, Special simple rings with involution, J. Algebra 6 (1967), 369375.

[13] I.N. Herstein, Rings with Involution, University of Chicago Press, Chicago, 1976.

[14] E. Jespers, M. Ruiz Marín: On symmetric elements and symmetric units in group rings, Comm. Algebra 34 (2006), 727-736.

[15] G.P. Kukin: Problem of equality and free products of Lie algebras and associative algebras, Sibirsk. Mar. Zh. 24 (1983), 85-96.

[16] G. Lee: Group rings whose symmetric elements are Lie nilpotent, Proc. Amer. Math. Soc. 127 (1999), 3153-3159.

[17] G. Lee: The Lie n-Engel property in group rings, Comm. Algebra 28 (2000), $867-881$.

[18] G. Lee: Group identities on units and symmetric units of group rings. Algebra and Applications, 12. Springer-Verlag London, 2010.

[19] G. Lee, S.K. Sehgal, E. Spinelli, Group algebras whose symmetric and skew elements are Lie solvable, Forum Math. 21 (2009) 661-671.

[20] G. Lee, S.K. Sehgal, E. Spinelli: Lie properties of symmetric elements in group rings. II, J. Pure Appl. Algebra 213 (2009), 1173-1178.

[21] D.S. Passman: Enveloping algebras satisfying a polynomial identity, J. Algebra 134 (1990), 469-490.

[22] V.M. Petrogradsky: Existence of identities in the restricted enveloping algebra, Math. Zametki 49 (1991), 84-93.

[23] D.M. Riley, A. Shalev: The Lie structure of enveloping algebras, J. Algebra 162 (1993), 46-61. 
[24] S. Siciliano: On the Lie algebra of skew-symmetric elements of an enveloping algebra, J. Pure Appl. Algebra 215 (2011), 72-76.

[25] I. Stewart: Infinite-dimensional Lie algebras in the spirit of infinite group theory, Compositio Mathematica 22 (1970), 313-331.

[26] H. Strade: Simple Lie algebras over fields of positive characteristic I. Structure theory. Walter de Gruyter \& Co., Berlin/New York, 2004.

[27] H. Strade, R. Farnsteiner: Modular Lie algebras and their representations, Marcel Dekker, New York, 1988.

[28] H. Usefi: Enveloping algebras of restricted Lie superalgebras satisfying nonmatrix polynomial identities, preprint vailable on arXiv:0910.2977.

Dipartimento di Matematica "E. De Giorgi", Università del Salento, Via Provinciale Lecce-Arnesano, 73100-Lecce, Italy

E-mail address: salvatore.siciliano@unisalento.it

Department of Mathematics, University of Toronto, Bahen Centre, 40 St. George St., Toronto, Ontario, Canada, M5S 2E4

E-mail address: usefi@math.toronto.edu 